\title{
Axial Magnetic Field Sensing for Pulsed Magnetic Flux Leakage Hairline Crack Detection and Quantification
}

\author{
Chukwunonso K. Okolo and Turgut Meydan \\ Wolfson Centre for Magnetics, School of Engineering \\ Cardiff University, \\ Cardiff, United Kingdom
}

\begin{abstract}
The Magnetic Flux Leakage (MFL) testing method is a well-established branch of electromagnetic non-destructive testing technology extensively used to observe, analyze and estimate the level of imperfections (cracks, corrosions, pits, dents, etc.) affecting the quality of ferromagnetic steel structures. However the conventional MFL (DCMFL) method are not capable of estimating the defect sizes and orientation, hence an additional transducer is required to provide the extra information needed. This paper takes the detection and quantification of tangentially oriented rectangular surface and far-surface hairline cracks as the research objective. It uses an optimized pulsed magnetic flux leakage probe system to establish the location and geometries of such cracks. The results gathered from the approach show that data using the axial $\left(B_{x}\right)$ field component can provide detailed locational information about hairline cracks especially the shape, size and orientation when positioned perpendicular to the applied field.
\end{abstract}

Keywords- defects, pulse magnetic flux leakage, Hall Effect sensor, pipeline, steel plates.

\section{INTRODUCTION}

Pipelines are used in oil and gas industries and they come with wall thickness ranging from $8 \mathrm{~mm}$ to $12 \mathrm{~mm}$ [1]. They are manufactured with precise specifications and requirements, to make sure the users, public and environment are safe. Over time external forces can impair the pipeline to a state capable of causing a spill or rupture. A crack is a flaw type caused by stress-induced separation of the material. It could develop from the rise and fall of the pipes operating pressure, which causes a slight change in the shape of the pipe [2]. The Magnetic Flux Leakage (MFL) technique, which was first carried out on storage tanks in 1988 by Saunderson, is a non-contact method of Non-Destructive Testing (NDT) used for locating and quantifying defects in ferromagnetic steel components [3]. The MFL technologies have been productively employed in the oil and gas industries for the inspection of: tubes, pipes, tank floors, and railway line confirmation. Recent improvements in MFL technology has helped to prevent serious damages such as breaking of pipelines, breakdown of reactors and wrecking of trains. The traditional MFL inspection technique involves the use of dc current for magnetization of the test sample. This method provides limited information about the defect based on its position and size, and it requires demagnetization. Also the defect has to occur on just one side of the test sample to ensure accurate deduction of the defect features, since the method depends solely on the intensity of the MFL signal.

The Pulsed Magnetic Flux Leakage (PMFL) technique is a state-of-the-art electromagnetic non-destructive evaluation method. It provides the advantage of using an excitation signal with a range of frequency components, which delivers the deeper penetration depth of low-frequency excitation and the sensitivity to surface measurements of high-frequency excitation [4]. One of the latest developments in the MFLNDT technology is the improvement from easy detection of flaws (qualitative assessment) to the evaluation of defect location and parameters (quantitative assessment) [4, 5]. Many MFL techniques have been presented by researchers working in the Quantitative non-destructive testing (QNDT) of materials [4-7]. However, the PMFL method has been proven to outperform conventional MFL methods in delivering useful quantitative data for estimating defect parameters. In addition to providing a wide spectrum of frequency components, which delivers deeper penetration depth when compared to the traditional MFL technique, information relating to the defect location and parameters can be established from features contained in the transient signal. The principal features needed to evaluate the size and depth information of the defect from the transient signal are the time-to-peak and the magnitude of the differential PML signals [8].

\section{PULSE MFL INSPECTION}

The PMFL inspection principle involves magnetizing the test sample (ferromagnetic steel pipe) with either a rectangular or square waveform (pulsed voltage), with a constant duty cycle, to near or complete saturation. A magnetic field is generated perpendicular to defect within the sample. If there is no defect, a greater proportion of the flux will flow through the sample. However, if there is a defect, then a defect field will be generated and the flux lines will change direction due to an increase in magnetic reluctance caused by a decrease in magnetic permeability at the defective region [9]. This will cause some of the flux to leak away from the material surface, and a leakage magnetic field will build up at the defective region. The leakage field can then be detected using an appropriate magnetic field sensor. The PML testing technique allows samples with large thicknesses to be inspected for farsurface flaws by providing the much desired deep 


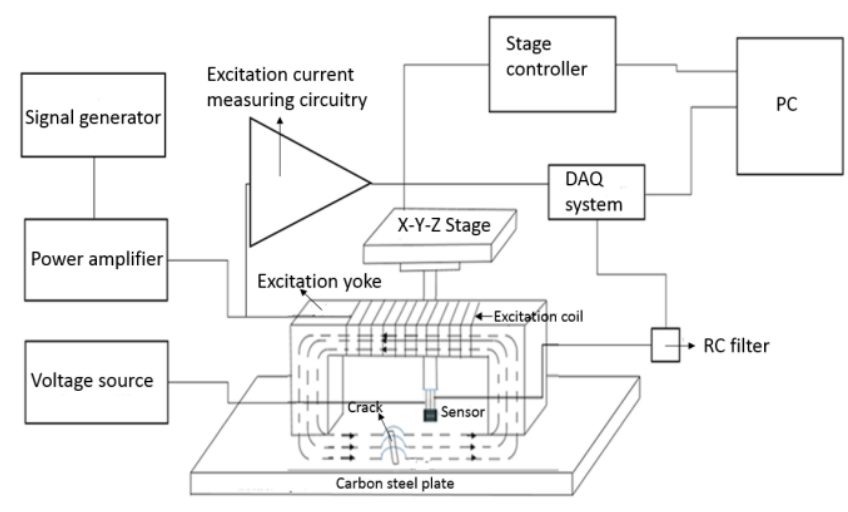

Fig. 1. Showing the PMFL experimental probe system set-up.

penetration depth, while still retaining a satisfactory sensitivity for surface and far-surface flaws. The penetration depth $d$ of the pulsed signal which corresponds to the time $t$ of the pulse transmitted in the test sample can be obtained from equation (1), where $\sigma$ and $\mu$ are the electrical conductivity and magnetic permeabilty of the test sample.

$$
d=\sqrt{\frac{t}{\pi \mu \sigma}}
$$

Also, further information such as the position, shape and parameters of the defect could be obtained from the PMFL signal.

\section{EXPERIMENTAL METHOD}

To explore the practicality of the experimental PMFL technique for tangential hairline crack detection and quantification, a 3D FEM software (Magnet 7.6 software by INFOLYTICA) has been used to model an optimized non-linear PMFL measurement system capable of detecting such cracks, prior to this work. The 3D model was used to optimize the magnetization and sensing methodology in order to improve the detection sensitivity of the PMFL probe system. The accuracy and practicality of the system has been assessed for the detection of surface and far-surface hairline cracks on $10 \mathrm{~mm}$ thick low carbon steel plate using an experimental approach. The dimension of the sample used is $350 \mathrm{~mm} \times 60 \mathrm{~mm} \times 10 \mathrm{~mm}$ with a conductivity of $1.17 \times 10^{7} \mathrm{~S} / \mathrm{m}$. The excitation yoke (silicon iron) used has leg height of $80 \mathrm{~mm}$, leg length of $30 \mathrm{~mm}$, leg width of $60 \mathrm{~mm}$, leg spacing of $240 \mathrm{~mm}$ with a conductivity of $2.17 \times 10^{6} \mathrm{~S} / \mathrm{m}$. The maximum relative magnetic permeability for the sample and yoke are 100 and 4000 respectively. The test set-up for the experimental investigation is shown in Fig. 1. The probe is driven with a square waveform, the excitation coil is fed with a $4 \mathrm{~V}$ amplitude voltage, $500 \mathrm{~ms}$ period, $50 \%$ duty cycle and the rise and fall time is set to $10 \mathrm{~ns}$. The positive full cycle of the MFL inspection system is analyzed and the distribution pattern of the axial $\left(B_{x}\right)$ component of the MFL signal is acquired for different pulse periods. However, the $500 \mathrm{~ms}$ period gave the best result. Fourteen different plate samples with varying crack depths and a constant crack width and length of $0.2 \mathrm{~mm}$ and $10 \mathrm{~mm}$ respectively, were tested. Measurements were made by scanning a single Hall Effect sensor (A1302KUA-T) within the crack area
$(24 \mathrm{~mm} \times 24 \mathrm{~mm})$ in steps of $0.1 \mathrm{~mm}$, with a constant sensor liftoff of $0.5 \mathrm{~mm}$. The sensor is positioned perpendicular to the orientation of the crack, and held in place by a 3D printed sensor holder attached to an $x-y-z$ translation stage system. The axial $\left(B_{x}\right)$ component of the PMFL signal is measured using the Hall sensor and a data acquisition system (NI USB-6366) with 16-bit analogue to digital conversion card is used to digitize the filtered output and stored in a computer for signal processing. A LabVIEW interface is used to visualize data and communicate with the motors and sensor electronics. For each scanning cycle, data were collected at 1000 samples per second.

\section{EXPERIMENTAL RESULTS AND DISCUSSIONS}

In order to extract the crack features from the PMFL signals, the method of first differential approach is used. The main idea is that supposing $c$ and $c_{0}$ are two samples with exact same magnetic properties and physical size, except that sample $c$ has a crack while $c_{0}$ has no crack. The differential crack signal $\Delta B_{x c}=B_{x c}-B_{x c_{0}}$ is acquired, where $B_{x c}$ is the crack signal and $B_{x c_{0}}$ is the reference signal. Fig. 2 shows the result obtained from an area scan performed in the vicinity of a $4 \mathrm{~mm}$ deep surface hairline crack. Fig. $2 \mathrm{a}$ and Fig. $2 \mathrm{~b}$ shows a typical crack signal and differential crack signal respectively. The behavior of the leakage signal in both cases is similar, both rising sharply as the PMFL sensor approaches the crack and falls as it moves away from it. However, the amplitude of the leakage field for the crack signal is higher than that of the differential crack signal. The signal to noise ratio $\operatorname{SNR}=20 \log \left(\frac{S}{N}\right)$ for this particular crack size is $34.8 \mathrm{~dB}$ Where $S$ is the amplitude of the PMFL signal obtained from a crack region, while $N$ is the PMFL signal obtained from a crack free region. Fig. 3 shows the crack and differential crack signals obtained at the midpoint of seven different surface hairline cracks with varying depth sizes. The PMFL signal amplitude was found to be strongly related to the crack depth. A small percentage change in crack depth caused a significant change in the $B_{x}$ field amplitude.

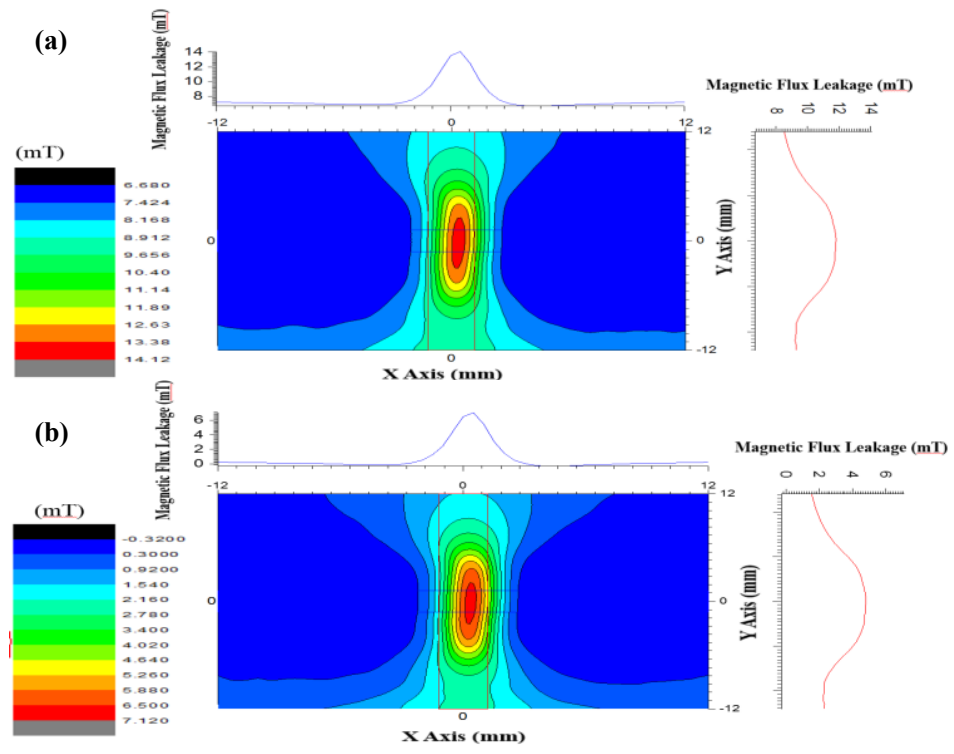

Fig. 2. Showing the measured $B_{x}$ component of the MFL signal from a $4 \mathrm{~mm}$ deep surface hairline crack (a) Crack signal and (b) Differential Crack signal. 


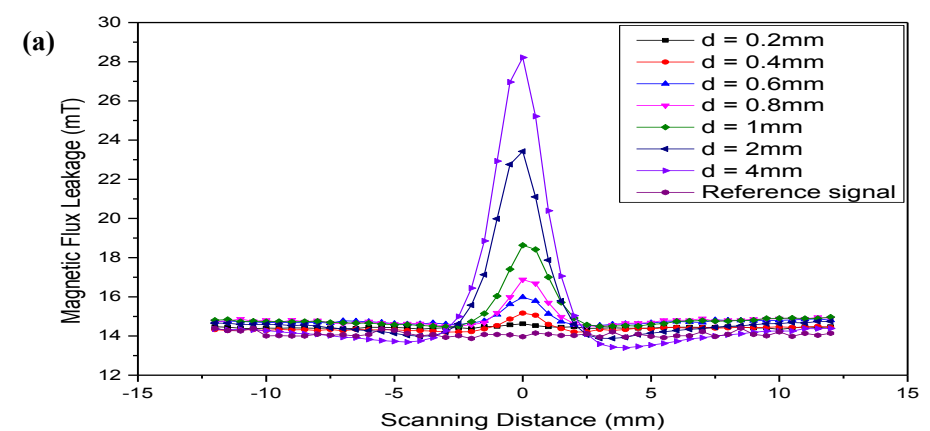

(b)

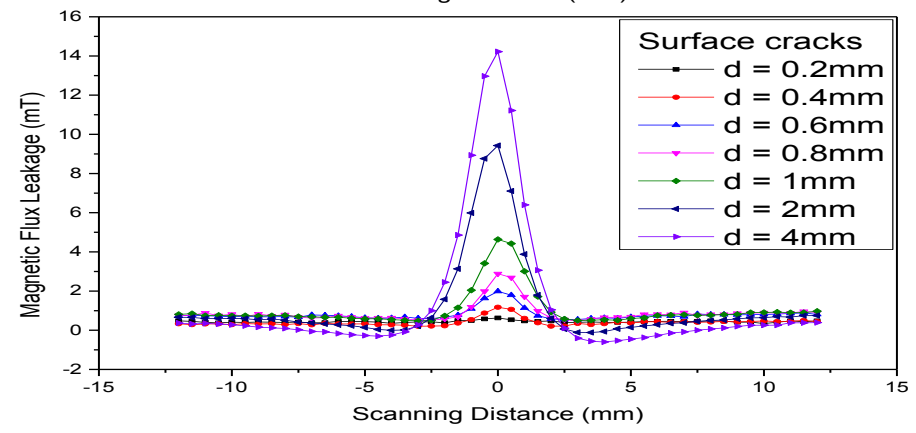

Fig. 3. Showing the measured $B_{x}$ component of the MFL signal from a $4 \mathrm{~mm}$ deep surface hairline crack at the crack centre (a) Crack signal and (b) Differential Crack signal.

Fig. 4 shows a comparison between the crack and differential crack signals for both the surface and far-surface hairline cracks with varying depth sizes. It can be seen that the amplitude of the PMFL signal increases as the crack depth increases from left to right, and the relationship between the intensity of leakage field signal and the crack depth is linearly dependent. Also it can be seen that the PMFL sensor is able to detect a $0.4 \mathrm{~mm}$ deep far-surface crack located $9.6 \mathrm{~mm}$ below the sample surface, with a SNR of $11 \mathrm{~dB}$. The approximate shape of cracks present in a pipeline structure could be obtained from the distribution pattern and amplitude of the PMFL signals. Fig. 2a and Fig. $2 b$ displays the signal patterns with respect to the sensing path distance. The width and length sizes of the crack can be estimated from the width of the differential crack signal along the width and length directions respectively, as shown in Fig.5. But it is difficult to estimate the depth of the crack from the signal width, since the signal width is seldom affected by the crack depth as can be inferred in Fig. 3.

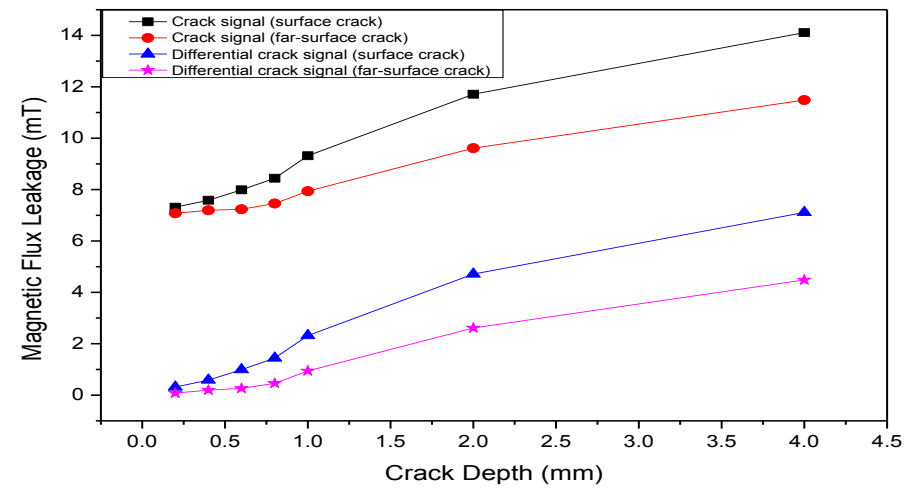

Fig. 4. Showing a comparison between the crack and differential crack signals for both the surface and far surface cracks.

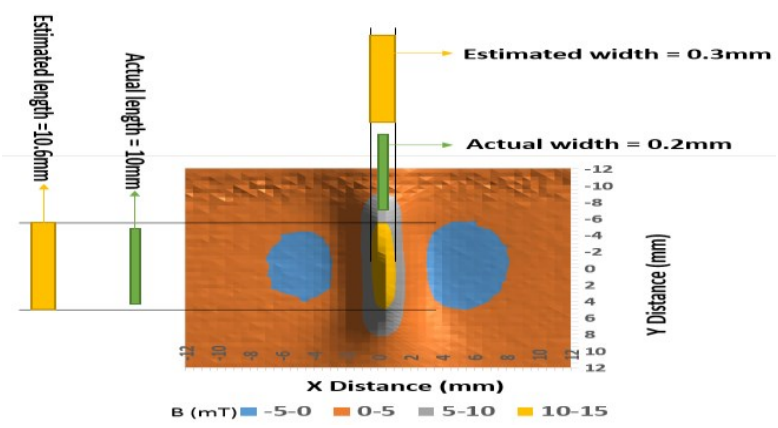

Fig. 5. Showing the estimation of crack width and length on the tangential and axial direction of the differential signal respectively, for a $4 \mathrm{~mm}$ deep surface hairline crack.

\section{CONCLUSION}

This paper has successfully employed the axial PMFL sensing technique, for the detection and quantification of tangentially oriented hairline surface and far-surface cracks in ferromagnetic steel pipelines. The PMFL inspection system was designed to generate an axial magnetic field in order to maximize the detectability of the leakage signal around the tangentially oriented cracks. The experimental findings show that the width and length of the crack can be estimated from the width of the differential crack signal along the width and length directions respectively. Also the PMFL signal amplitude was found to be strongly dependent on the crack depth and a linear relationship exists between the crack depth and the leakage field intensity. The optimized system was able to enhance the detection sensitivity of the PMFL inspection significantly by detecting a $0.2 \mathrm{~mm}$ deep ( $2 \%$ wall loss) surface hairline crack and a $0.4 \mathrm{~mm}$ deep far-surface hairline crack with a penetration depth of $9.6 \mathrm{~mm}$.

\section{REFERENCES}

[1] M. Boat, N. Pearson, R. Lieb, J. Davies, R. James and B. Woodhead, "Factors that affect the defect sizing capabilities of the magnetic flux leakage technique", Silverwing (UK) Ltd, 2012.

[2] U.S Department of Transportation. 2011. Pipe defects and anomalies (pipeline \& safety administration). [Online]. Available at: http://primis.phmsa.dot.gov/comm/FactSheets/FSPipeDefects.htm?noca che $=9047$. [Accessed 18 November 16].

[3] D H Saunderson, 'The MFE tank floor scanner - a case history', IEEE Colloquium on Non-Destructive Evaluation, 1988.

[4] Wilson, John W., and Gui Yun Tian. "Pulsed Electromagnetic Methods For Defect Detection And Characterisation". NDT \& E International 40.4 (2007): pp. 275-283.

[5] L. Yong, J. Wilson, and G. Y. Tian. "Experiment and Simulation Study of 3D Magnetic Field Sensing For Magnetic Flux Leakage Defect Characterisation". NDT \& E International 40.2 (2007): pp. 179-184.

[6] V. Suresh and A. Abudhahir, "An Analytical model for Prediction of Magnetic Flux Leakage from Surface Defects in Ferromagnetic Tubes". Measurement Science Review, 16, (2016), No. 1, pp. 8-13.

[7] Y. Sun, and Y. Kang. "A New MFL Principle And Method Based On Near-Zero Background Magnetic Field". NDT \& E International 43.4 (2010): pp. 348-353.

[8] Y. Tang, M. Pan C. F. Luo, "Feature extraction Based on the Principal Component Analysis for Pulsed Magnetic Flux Leakage Testing". International Conference on Mechatronic Science, Electric Engineering and Computer August 19-22, 2011, Jilin, China.

[9] Shi, Yan et al. "Theory and application of magnetic flux leakage pipeline detection". Sensors 15.12 (2015): pp. 31036-31055. 